\title{
HARMONIC OPERATORS OF ERGODIC QUANTUM GROUP ACTIONS
}

\author{
MASSOUD AMINI, MEHRDAD KALANTAR, AND MOHAMMAD S. M. MOAKHAR
}

(Communicated by Marius Junge)

\begin{abstract}
In this paper we study the harmonic elements of (convolution) Markov maps associated to (ergodic) actions of locally compact quantum groups on ( $\sigma$-finite) von Neumann algebras. We give several equivalent conditions under which the harmonic elements are trivial.
\end{abstract}

\section{INTRODUCTION AND PRELIMINARIES}

The noncommutative Poisson boundary, i.e., the space of fixed points of a normal unital completely positive map on a von Neumann algebra, has been extensively studied in the last few decades, both in general and concrete settings [3, [], [1], [9], 2]. These boundaries are of particular interest for the class of Markov maps associated to (homogeneous) Markov processes on algebraic structures, such as the convolution maps of probability measures on locally compact (quantum) groups.

In [8] Izumi introduced noncommutative random walks on discrete quantum groups and studied the associated Poisson boundaries. In this paper we consider a more general setup. The Markov operators whose Poisson boundaries are investigated here arise from the convolution action of quantum probability measures on locally compact quantum groups on von Neumann algebras.

To further clarify the setup and motivate the discussion, let us consider the classical case: Let $G$ be a locally compact group, acting measurably on a $\sigma$-finite probability space $(X, \nu)$. When $\nu$ is quasi-invariant, $G$ acts canonically on $L^{\infty}(X, \nu)$, and for a probability measure $\mu$ on $G$ one may define a convolution map on $L^{\infty}(X, \nu)$ by

$$
\mu \star h(x):=\int_{X} h(g x) d \mu(g) \quad h \in L^{\infty}(X, \nu), \quad x \in X .
$$

This convolution map defines a Markov operator, and therefore a (time independent) Markov chain on $X$ with transition probabilities

$$
p(x, A)=\mu(\{g \in G: g x \in A\}) \quad x \in X, A \subseteq X .
$$

A function $h \in L^{\infty}(X, \nu)$ is called $\mu$-harmonic if it is fixed under convolution by $\mu$, i.e.,

$$
\int_{X} h(g x) d \mu(g)=h(x) \quad \text { for } \nu \text {-a.e. } x \in X \text {. }
$$

Received by the editors January 27, 2014.

2010 Mathematics Subject Classification. Primary 46L89, 46L55; Secondary 46L07, 22D25. 
The space of all $\mu$-harmonic functions is isomorphic, through the Poisson transformation, to the space of all essentially bounded measurable functions on the Poisson boundary of the associated Markov chain on $X[11$.

In this paper we investigate harmonic elements of the convolution maps associated to quantum group actions. Convolution Markov operators on $G$-spaces are studied in the classical setting (cf. [13, [6, [7]), but some of our results here on harmonic functions are new even for group actions. A more detailed study of the classical convolution Markov chains on transformation groups is done in [16].

Let us recall basic definitions and set the terminology used in this paper. For more details on locally compact quantum groups and their actions we refer the reader to [17] and [20].

A locally compact quantum group $\mathbb{G}$ is a quadruple $\left(\mathcal{L}^{\infty}(\mathbb{G}), \Gamma, \varphi, \psi\right)$, where $\mathcal{L}^{\infty}(\mathbb{G})$ is a von Neumann algebra, $\Gamma: \mathcal{L}^{\infty}(\mathbb{G}) \rightarrow \mathcal{L}^{\infty}(\mathbb{G}) \bar{\otimes} \mathcal{L}^{\infty}(\mathbb{G})$ is a co-associative comultiplication, and $\varphi$ and $\psi$ are the (normal faithful semi-finite) left and right Haar weights on $\mathcal{L}^{\infty}(\mathbb{G})$, respectively. The corresponding GNS Hilbert spaces $L^{2}(\mathbb{G}, \varphi)$ and $L^{2}(\mathbb{G}, \psi)$ are isomorphic and are denoted by the same notation $\mathcal{L}^{2}(\mathbb{G})$. The (left) fundamental unitary $W$ of $\mathbb{G}$ is a unitary operator on $\mathcal{L}^{2}(\mathbb{G}) \otimes \mathcal{L}^{2}(\mathbb{G})$, satisfying the pentagonal equation that implements the comultiplication $\Gamma$.

The reduced quantum group $C^{*}$-algebra $\mathcal{C}_{0}(\mathbb{G})={\overline{\left\{(\iota \otimes \omega) W: \omega \in B(H)_{*}\right.}}^{\|\cdot\|}$ is a weak* dense $C^{*}$-subalgebra of $\mathcal{L}^{\infty}(\mathbb{G})$. Let $\mathcal{L}^{1}(\mathbb{G})$ be the predual of $\mathcal{L}^{\infty}(\mathbb{G})$. The pre-adjoint of $\Gamma$ induces an associative completely contractive multiplication

$$
\star: f \otimes g \in \mathcal{L}^{1}(\mathbb{G}) \widehat{\otimes} \mathcal{L}^{1}(\mathbb{G}) \rightarrow f \star g=(f \otimes g) \Gamma \in \mathcal{L}^{1}(\mathbb{G})
$$

on $\mathcal{L}^{1}(\mathbb{G})$. Also for the operator dual $\mathcal{M}(\mathbb{G})$ of $C_{0}(\mathbb{G})$, there exists a completely contractive multiplication on $\mathcal{M}(\mathbb{G})$ given by the convolution

$$
\star: \mu \otimes \nu \in \mathcal{M}(\mathbb{G}) \widehat{\otimes} \mathcal{M}(\mathbb{G}) \mapsto \mu \star \nu:=\mu(\iota \otimes \nu) \Gamma=\nu(\mu \otimes \iota) \Gamma \in \mathcal{M}(\mathbb{G})
$$

such that $\mathcal{M}(\mathbb{G})$ contains $\mathcal{L}^{1}(\mathbb{G})$ as a norm closed two-sided ideal. In particular, every $\mu \in \mathcal{M}(\mathbb{G})$ induces a convolution map $\mathcal{L}^{1}(\mathbb{G}) \ni \omega \mapsto \mu \star \omega \in \mathcal{L}^{1}(\mathbb{G})$ on $\mathcal{L}^{1}(\mathbb{G})$. We denote the adjoint of the latter by $\Phi^{\mu}$, which is a normal completely bounded map on $\mathcal{L}^{\infty}(\mathbb{G})$. When $\mu$ is a state, $\Phi^{\mu}$ is a Markov map, i.e., it is also unital and completely positive.

For a von Neumann algebra $N$, a (left) action $\alpha: \mathbb{G} \curvearrowright N$ of $\mathbb{G}$ on $N$ is an injective $*$-homomorphism $\alpha: N \rightarrow \mathcal{L}^{\infty}(\mathbb{G}) \bar{\otimes} N$ such that

$$
(\Gamma \otimes \iota) \alpha=(\iota \otimes \alpha) \alpha .
$$

The action $\alpha$ is called ergodic if the fixed point algebra is trivial, that is,

$$
N^{\alpha}:=\{x \in N: \alpha(x)=1 \otimes x\}=\mathbb{C} 1 .
$$

\section{Convolution operators on $\mathbb{G}$-Spaces}

For $\mu \in \mathcal{M}(\mathbb{G})$ by [14, Theorem 2.1] one obtains a (unique) normal completely bounded map $\Phi_{\alpha}^{\mu}$ on $N$ such that

$$
\alpha \Phi_{\alpha}^{\mu}=\left(\Phi^{\mu} \otimes \iota\right) \alpha .
$$

When $\mu \in \mathcal{L}^{1}(\mathbb{G})$, we have $\Phi_{\alpha}^{\mu}=(\mu \otimes \iota) \alpha$. 
We denote by $\mathcal{P}(\mathbb{G})$ the set of all states $\mu$ in $\mathcal{M}(\mathbb{G})$. For $\mu \in \mathcal{P}(\mathbb{G})$, the corresponding operator $\Phi_{\alpha}^{\mu}$ is a Markov map on $N$. We denote by $\mathcal{H}_{\alpha}^{\mu}$ the space of $\mu$-harmonic elements in $N$, i.e., the space of fixed points of the Markov operator $\Phi_{\alpha}^{\mu}$. This is a weak ${ }^{*}$ closed operator system in $N$, and if we choose a free ultrafilter $\mathcal{U}$ on $\mathbb{N}$, we obtain an unital completely positive idempotent map $E_{\alpha}^{\mu}$ on $N$,

$$
E_{\alpha}^{\mu}(x)=\text { weak }^{*}-\lim _{\mathcal{U}} \frac{1}{n} \sum_{k=1}^{n}\left(\Phi_{\alpha}^{\mu}\right)^{k}(x) .
$$

This allows us to define a von Neumann algebra structure on the operator system $\mathcal{H}_{\alpha}^{\mu}$ via the Choi-Effros product $x \circ y:=E_{\alpha}^{\mu}(x y)$ (cf. [3]).

One may consider the co-multiplication $\Gamma$ as a (left) action of $\mathbb{G}$ on $\mathcal{L}^{\infty}(\mathbb{G})$. The following result describes $\mathcal{H}_{\alpha}^{\mu}$ for $\alpha: \mathbb{G} \curvearrowright N$ in terms of $\mathcal{H}_{\Gamma}^{\mu}$.

Proposition 2.1. Let $\alpha: \mathbb{G} \curvearrowright N$ be an action of a locally compact quantum group $\mathbb{G}$ on a von Neumann algebra $N$, and let $\mu \in \mathcal{P}(\mathbb{G})$. Then

$$
\mathcal{H}_{\alpha}^{\mu}=\left\{x \in N: \alpha(x) \in \mathcal{H}_{\Gamma}^{\mu} \bar{\otimes} N\right\} .
$$

Proof. The inclusion $\supseteq$ follows from (2.1) and injectivity of $\alpha$. For the reverse inclusion, suppose that $x \in \mathcal{H}_{\alpha}^{\mu}$, i.e., $\Phi_{\alpha}^{\mu}(x)=x$. Then using (2.1) we obtain

$\Phi^{\mu}((\iota \otimes \omega) \alpha(x))=(\iota \otimes \omega)\left(\left(\Phi^{\mu} \otimes \iota\right) \alpha(x)\right)=(\iota \otimes \omega)\left(\alpha\left(\Phi_{\alpha}^{\mu}(x)\right)\right)=(\iota \otimes \omega) \alpha(x)$

for all $\omega \in N_{*}$. Hence $(\iota \otimes \omega) \alpha(x) \in \mathcal{H}_{\Gamma}^{\mu}$ for all $\omega \in N_{*}$ and therefore $\alpha(x) \in$ $\mathcal{H}_{\Gamma}^{\mu} \otimes_{\mathcal{F}} N$, where $\otimes_{\mathcal{F}}$ denotes the Fubini tensor product (cf. [5]). Since $\mathcal{H}_{\Gamma}^{\mu}$ is a von Neumann algebra with its Choi-Effros product, it follows from [19, Proposition 3.3] that $\mathcal{H}_{\Gamma}^{\mu} \otimes_{\mathcal{F}} N=\mathcal{H}_{\Gamma}^{\mu} \bar{\otimes} N$, and the inclusion follows.

Corollary 2.2. Let $\alpha: \mathbb{G} \curvearrowright N$ be an ergodic action of a locally compact quantum group $\mathbb{G}$ on a von Neumann algebra $N$, and let $\mu \in \mathcal{P}(\mathbb{G})$. If $\mathcal{H}_{\Gamma}^{\mu}=\mathbb{C} 1$, then $\mathcal{H}_{\alpha}^{\mu}=\mathbb{C} 1$.

Proof. Let $x \in \mathcal{H}_{\alpha}^{\mu}$. By the above proposition, there exists $y \in N$ such that $\alpha(x)=1 \otimes y$. Therefore

$$
1 \otimes \alpha(y)=(\iota \otimes \alpha) \alpha(x)=(\Gamma \otimes \iota) \alpha(x)=(\Gamma \otimes \iota)(1 \otimes y)=1 \otimes 1 \otimes y,
$$

which implies $y \in \mathbb{C} 1$, by ergodicity of $\alpha$, hence $x \in \mathbb{C} 1$.

For $\omega \in N_{*}$, we define a completely bounded map $\phi_{\omega}: N \rightarrow \mathcal{L}^{\infty}(\mathbb{G})$ by $\phi_{\omega}(x)=$ $(\iota \otimes \omega) \alpha(x)$. Then the following are easily derived

$$
\left(\iota \otimes \phi_{\omega}\right) \alpha=\Gamma \circ \phi_{\omega} \quad \text { and } \quad \Phi^{\mu} \circ \phi_{\omega}=\phi_{\mu \star \omega},
$$

for all $\omega \in N_{*}$ and $\mu \in \mathcal{M}(\mathbb{G})$, where $\mu \star \omega:=\left(\Phi_{\alpha}^{\mu}\right)_{*}(\omega)$.

An essential fact behind many of the results concerning the convolution maps in the quantum setting is that for any $\mu \in \mathcal{P}(\mathbb{G})$, the convolution map $\Phi^{\mu}$ is a faithful map on $\mathcal{L}^{\infty}(\mathbb{G})$. This follows easily from the faithfulness of the Haar weight, and its invariance under convolution maps (cf. [15, Lemma 3.4]). This, together with (2.1), yield that for any action $\alpha: \mathbb{G} \curvearrowright N$ and $\mu \in \mathcal{P}(\mathbb{G})$, the map $\Phi_{\alpha}^{\mu}$ is faithful on $N$. But, in the absence of a "Haar weight" for a general action $\alpha: \mathbb{G} \curvearrowright N$, the analogous result for the map $\phi_{\omega}$ is by no means trivial. 
Theorem 2.3. Let $\alpha: \mathbb{G} \curvearrowright N$ be an action of a locally compact quantum group $\mathbb{G}$ on a $\sigma$-finite von Neumann algebra $N$. Then the following are equivalent:

(1) the action $\alpha$ is ergodic;

(2) for any nonzero $\omega \in N_{*}^{+}$, the map $\phi_{\omega}: N \longrightarrow \mathcal{L}^{\infty}(\mathbb{G})$ is faithful.

Proof. $(2) \Rightarrow(1)$ : Suppose $\alpha$ is not ergodic. Let $e \in N^{\alpha}$ be a nontrivial projection, and let $\omega \in N_{*}$ be a normal state whose support projection is $e$. Then we have

$$
\phi_{\omega}(1-e)=(\iota \otimes \omega) \alpha(1-e)=\langle\omega, 1-e\rangle 1=0 .
$$

$(1) \Rightarrow(2)$ : Let $\omega \in N_{*}^{+}$be nonzero, and suppose $x \in N^{+}$is such that $\phi_{\omega}(x)=0$. Denote by $p$ the support projection of $\omega$. Then for every $\rho \in \mathcal{L}^{1}(\mathbb{G})$ we have $\omega((\rho \otimes \iota) \alpha(x))=0$. This implies $(\rho \otimes \iota)(\alpha(x)(1 \otimes p))=0$ for all $\rho \in \mathcal{L}^{1}(\mathbb{G})$, and therefore $\alpha(x)(1 \otimes p)=0$. Now consider the left ideal

$$
M:=\{y \in N: \alpha(y)(1 \otimes p)=0\},
$$

and suppose $q \in N$ is a projection such that $M=N q$. Then for every $\rho \in \mathcal{L}^{1}(\mathbb{G})$ we obtain

$$
\alpha((\rho \otimes \iota) \alpha(q))(1 \otimes p)=\left(\Phi^{\rho} \otimes \iota\right)(\alpha(q)(1 \otimes p))=0,
$$

which shows that $(\rho \otimes \iota) \alpha(q) \in M$, whence $[(\rho \otimes \iota) \alpha(q)] q=(\rho \otimes \iota) \alpha(q)$ for all $\rho \in \mathcal{L}^{1}(\mathbb{G})$. This implies $\alpha(q)(1 \otimes q)=\alpha(q)$, i.e.,

$$
\alpha(q) \leq 1 \otimes q .
$$

Denote $q^{\prime}=1-q$, and let $\theta$ be a normal faithful state on $N$ with the GNS map $\Lambda_{\theta}: N \rightarrow H_{\theta}$. Let $\tilde{\theta}$ be the dual weight on the crossed product $\mathbb{G}_{\alpha} \ltimes N$ (cf. [20, Definition 3.1]), with modular operator and modular conjugation $\nabla_{\tilde{\theta}}$ and $J_{\tilde{\theta}}$. Let and $\hat{\nabla}$ and $\hat{J}$ be the modular operator and modular conjugation of the Haar weight $\hat{\varphi}$ of the dual quantum group $\hat{\mathbb{G}}$. Then, for every $y \in N$ and $\xi \in \mathcal{D}\left(\hat{\nabla}^{\frac{1}{2}}\right)$ we have

$$
\begin{aligned}
\alpha\left(q^{\prime}\right) & (1 \otimes q)\left(\hat{J} \hat{\nabla}^{\frac{1}{2}} \xi \otimes \Lambda_{\theta}(y)\right) \\
& =\alpha\left(q^{\prime}\right)\left(\hat{J} \hat{\nabla}^{\frac{1}{2}} \xi \otimes \Lambda_{\theta}(q y)\right) \\
& =J_{\tilde{\theta}} \nabla_{\tilde{\theta}}^{\frac{1}{2}} \alpha\left(y^{*} q\right)\left(\xi \otimes \Lambda_{\theta}\left(q^{\prime}\right)\right) \quad(\text { by [20, Lemma 3.11] }) \\
& =J_{\tilde{\theta}} \nabla_{\tilde{\theta}}^{\frac{1}{2}} \alpha\left(y^{*}\right) \alpha(q)\left(1 \otimes q^{\prime}\right)\left(\xi \otimes \Lambda_{\theta}\left(q^{\prime}\right)\right) \\
& =0 \quad\left(\text { since } \alpha(q)\left(1 \otimes q^{\prime}\right)=0 \text { by (2.4) }\right) .
\end{aligned}
$$

Hence $\alpha\left(q^{\prime}\right)(1 \otimes q)=0$, which then together with (2.4) implies that $\alpha(q)=1 \otimes q$. Since $\alpha$ is ergodic, we conclude that $q=0$ or $q=1$. But $q=1$ implies $\omega=0$, so it follows that $q=0$, and in particular $x=0$.

Now, using the above theorem, we can prove a quantum version of the Maximum Principle, which clears the path to generalize a number of results on the triviality of certain classes of harmonic functions. For this, we need to impose a (rather weak) restriction on the measures $\mu$ we consider. We prove a Maximum Principle for spread-out quantum probability measures. The measure $\mu \in \mathcal{P}(\mathbb{G})$ is called 
spread out if there are $n \in \mathbb{N}$ and $0 \neq \omega \in \mathcal{L}^{1}(\mathbb{G})^{+}$such that $\omega \leq \mu^{n}$, or equivalently, if $\mu^{n}=\omega_{a}+\omega_{s}$, for some $n \in \mathbb{N}$, where $0 \neq \omega_{a} \in \mathcal{L}^{1}(\mathbb{G})^{+}$and $\omega_{s} \in \mathcal{M}(\mathbb{G})^{+}$.

Note that this is not a very restrictive condition. In the classical setting of locally compact groups, in order to have a well-defined notion of measure-theoretical boundaries, one has to restrict to such probability measures. For discrete (quantum) groups, every (quantum) measure is spread out. Also the assumption that $\mu$ is spread out is not necessary in the following lemma (and the results that follow) if $\mathbb{G}$ is co-amenable (which includes the case of locally compact groups). On the other hand, such a restriction is expected as we work in the very general setting of measurable actions and do not impose any continuity condition on the action.

Recall that $\mu \in \mathcal{P}(\mathbb{G})$ is nondegenerate if for every nonzero positive $x \in \mathcal{C}_{0}(\mathbb{G})$ there is $n \in \mathbb{N}$ such that $\left\langle x, \mu^{n}\right\rangle>0$.

Lemma 2.4 (The Maximum Principle). Let $\alpha: \mathbb{G} \curvearrowright N$ be an ergodic action of a locally compact quantum group $\mathbb{G}$ on a $\sigma$-finite von Neumann algebra $N$, and let $\mu \in \mathcal{P}(\mathbb{G})$ be a nondegenerate spread-out state. Suppose that $x \in \mathcal{H}_{\alpha}^{\mu}$ is a selfadjoint element which attains its norm on the set of normal states on $N$. Then $x \in \mathbb{C} 1$.

Proof. Suppose $x \in \mathcal{H}_{\alpha}^{\mu}$ is self-adjoint with $\|x\|=1$ (in particular $1-x \geq 0$ ), and suppose $\nu$ is a normal state on $N$ such that $\langle\nu, x\rangle=1$. Let $\mu \in \mathcal{P}(\mathbb{G})$ be nondegenerate and spread out. Define $\rho:=\sum_{n=1}^{\infty} \frac{1}{2^{n}} \mu^{n} \in \mathcal{P}(\mathbb{G})$. Since $\mu$ is spread out, we have $\rho=\omega_{a}+\omega_{s}$, where $0 \neq \omega_{a} \in \mathcal{L}^{1}(\mathbb{G})^{+}$and $\omega_{s} \in \mathcal{M}(\mathbb{G})^{+}$. Moreover, since $\mu$ is nondegenerate, $\rho$ is faithful on $\mathcal{C}_{0}(\mathbb{G})$ and extends uniquely to a strictly continuous faithful state on the multiplier $C^{*}$-algebra $\mathcal{C}(\mathbb{G})$ of $\mathcal{C}_{0}(\mathbb{G})$. Therefore $\omega_{a} \star \rho \in \mathcal{L}^{1}(\mathbb{G})^{+}$is faithful on $\mathcal{L}^{\infty}(\mathbb{G})$, and

$$
\begin{aligned}
0 \leq\left\langle\omega_{a} \star \rho, \phi_{\nu}(1-x)\right\rangle & =\left\langle\nu, \Phi_{\alpha}^{\omega_{a} \star \rho}(1-x)\right\rangle=\left\langle\nu, \Phi_{\alpha}^{\omega_{a}} \Phi_{\alpha}^{\rho}(1-x)\right\rangle \\
& =\left\langle\nu, \Phi_{\alpha}^{\omega_{a}}(1-x)\right\rangle \leq\left\langle\nu, \Phi_{\alpha}^{\rho}(1-x)\right\rangle \\
& =\langle\nu, 1-x\rangle=0 .
\end{aligned}
$$

By Theorem 2.3, the map $\phi_{\nu}$ is faithful, hence $1-x=0$.

Proposition 2.5. Let $\alpha: \mathbb{G} \curvearrowright N$ be an ergodic action of a locally compact quantum group $\mathbb{G}$ on a $\sigma$-finite von Neumann algebra $N$, and let $\mu \in \mathcal{P}(\mathbb{G})$ be nondegenerate and spread out. Then the following are equivalent:

(1) every normal state on $\mathcal{H}_{\alpha}^{\mu}$ can be extended to a normal state on $N$;

(2) $\mathcal{H}_{\alpha}^{\mu}=\mathbb{C} 1$.

Proof. We just need to prove $(1) \Rightarrow(2)$. Consider $\mathcal{H}_{\alpha}^{\mu}$ with its von Neumann algebra structure, and let $p \in \mathcal{H}_{\alpha}^{\mu}$ be a projection. Then $p$ attains its norm on a normal state on $\mathcal{H}_{\alpha}^{\mu}$, which, by the assumption, can be extended to a normal state on $N$. Hence $p \in \mathbb{C} 1$, by Lemma 2.4, therefore $\mathcal{H}_{\alpha}^{\mu}=\mathbb{C} 1$.

The following corollaries are then immediate.

Corollary 2.6. Let $\alpha: \mathbb{G} \curvearrowright N$ be an ergodic action of a locally compact quantum group $\mathbb{G}$ on a $\sigma$-finite von Neumann algebra $N$, and let $\mu \in \mathcal{P}(\mathbb{G})$ be nondegenerate and spread out. If the map $E_{\alpha}^{\mu}: N \rightarrow \mathcal{H}_{\alpha}^{\mu}$ is normal, then $\mathcal{H}_{\alpha}^{\mu}=\mathbb{C} 1$. 
Corollary 2.7. Let $\alpha: \mathbb{G} \curvearrowright N$ be an ergodic action of a locally compact quantum group $\mathbb{G}$ on a $\sigma$-finite von Neumann algebra $N$, and let $\mu \in \mathcal{P}(\mathbb{G})$ be nondegenerate and spread out. If $\mathcal{H}_{\alpha}^{\mu}$ is a subalgebra of $N$, then $\mathcal{H}_{\alpha}^{\mu}=\mathbb{C} 1$.

We close the section by proving a result on the multiplicative structure of $\mathcal{H}_{\alpha}^{\mu}$. The following proposition shows that $\mathcal{H}_{\alpha}^{\mu}$ can not contain any nontrivial *subalgebra of $N$.

Proposition 2.8. Let $\alpha: \mathbb{G} \curvearrowright N$ be an ergodic action of a locally compact quantum group $\mathbb{G}$ on a $\sigma$-finite von Neumann algebra $N$, and let $\mu \in \mathcal{P}(\mathbb{G})$ be nondegenerate and spread out. If $x \in \mathcal{H}_{\alpha}^{\mu}$ is such that $x x^{*}, x^{*} x \in \mathcal{H}_{\alpha}^{\mu}$, then $x \in \mathbb{C} 1$.

Proof. By the assumption we have $\Phi_{\alpha}^{\mu}\left(x^{*} x\right)=x^{*} x=\Phi_{\alpha}^{\mu}\left(x^{*}\right) \Phi_{\alpha}^{\mu}(x)$. Hence, by [5. Corollary 5.2.2], $\Phi_{\alpha}^{\mu}(y x)=\Phi_{\alpha}^{\mu}(y) \Phi_{\alpha}^{\mu}(x)$, for all $y \in N$. Similarly, we have $y x^{*} \in \mathcal{H}_{\alpha}^{\mu}$, for all $y \in \mathcal{H}_{\alpha}^{\mu}$. Hence, the weak* closed subalgebra generated by $1, x, x^{*}$ (denoted by $\mathcal{A}$ ) in $N$ is contained in $\mathcal{H}_{\alpha}^{\mu}$. Now, let $p \in \mathcal{A}$ be a nonzero projection (in the von Neumann algebra structure of $\mathcal{A}$ inherited from $N$ ), since $p$ attains its norm on a normal state on $N$, it follows from Lemma 2.4 that $p=1$, therefore $\mathcal{A}=\mathbb{C} 1$. In particular, $x \in \mathbb{C} 1$.

\section{TRiviality of CERTAin ClASSES OF HARMONIC ELEMENTS}

Having the Maximum Principle at our disposal, we can prove the following result, similar to [15. Theorem 3.7]. We denote by $\mathcal{K}(H)$ the space of all compact operators on a Hilbert space $H$.

Theorem 3.1. Let $\alpha: \mathbb{G} \curvearrowright N$ be an ergodic action of a locally compact quantum group $\mathbb{G}$ on a $\sigma$-finite von Neumann algebra $N$. If $\mu \in \mathcal{P}(\mathbb{G})$ is nondegenerate and spread out and $N$ acts on a Hilbert space $H$, then $\mathcal{H}_{\alpha}^{\mu} \cap \mathcal{K}(H) \subseteq \mathbb{C} 1$.

Proof. Let $x \in \mathcal{H}_{\alpha}^{\mu} \cap \mathcal{K}(H)$ be self-adjoint. Then there exists a state $\omega \in B(H)_{*}$ such that $|\langle\omega, x\rangle|=\|x\|$. Hence the restriction of $\omega$ to $N$ is a normal state on $N$ that realizes $\|x\|$. Therefore $x \in \mathbb{C} 1$ by Lemma 2.4. Since $\mathcal{H}_{\alpha}^{\mu} \cap \mathcal{K}(H)$ is generated by its self-adjoint elements, the result follows.

Corollary 3.2. Let $\alpha: \mathbb{G} \curvearrowright N$ be an ergodic action of a locally compact quantum group $\mathbb{G}$ on a finite dimensional von Neumann algebra $N$. Then $\mathcal{H}_{\alpha}^{\mu}=\mathbb{C} 1$ for any nondegenerate spread-out $\mu \in \mathcal{P}(\mathbb{G})$.

In the case of the action of a locally compact (quantum) group $\mathbb{G}$ on itself via the comultiplication, there is always a natural action of $\mathbb{G}$ on its Poisson boundaries. This follows from the fact that the comultiplication can be regarded both as a left or a right action of $\mathbb{G}$ on itself that commutes with each other. As shown by the following theorem, in the general setting of quantum group actions, where such two sided commuting actions do not exist, we cannot define a natural action of $\mathbb{G}$ on the corresponding Poisson boundaries.

Theorem 3.3. Let $\alpha$ be an ergodic action of a locally compact quantum group $\mathbb{G}$ on a von Neumann algebra $N$, and let $\mu \in \mathcal{P}(\mathbb{G})$ be nondegenerate and spread out. If $(\omega \otimes \iota) \alpha\left(\mathcal{H}_{\alpha}^{\mu}\right) \subseteq \mathcal{H}_{\alpha}^{\mu}$ for every $\omega \in \mathcal{L}^{1}(\mathbb{G})$, then $\mathcal{H}_{\alpha}^{\mu}=\mathbb{C} 1$.

Proof. As in the proof of Proposition 2.1 we conclude that $\alpha(x) \in \mathcal{L}^{\infty}(\mathbb{G}) \otimes_{\mathcal{F}} \mathcal{H}_{\alpha}^{\mu}=$ $\mathcal{L}^{\infty}(\mathbb{G}) \bar{\otimes} \mathcal{H}_{\alpha}^{\mu}$, for all $x \in \mathcal{H}_{\alpha}^{\mu}$. Hence the restriction of $\alpha$ to $\mathcal{H}_{\alpha}^{\mu}$ induces an ergodic action $\beta: \mathbb{G} \curvearrowright \mathcal{H}_{\alpha}^{\mu}$. Therefore $\Phi_{\beta}^{\mu}=\left.\Phi_{\alpha}^{\mu}\right|_{\mathcal{H}_{\alpha}^{\mu}}$, which implies that $\mathcal{H}_{\beta}^{\mu}=\mathcal{H}_{\alpha}^{\mu}$. Hence $\mathcal{H}_{\alpha}^{\mu}=\mathbb{C} 1$, by Corollary 2.7 . 
Corollary 3.4. Let $\mathbb{G}$ be a locally compact quantum group. If there exists a nondegenerate spread-out $\mu \in \mathcal{P}(\mathbb{G})$ in the center of the Banach algebra $\mathcal{M}(\mathbb{G})$, then $\mathcal{H}_{\alpha}^{\mu}=\mathbb{C} 1$, and $\mathbb{G}$ is amenable.

Proof. If $\mu$ is in the center of $\mathcal{M}(\mathbb{G})$, then for every $x \in \mathcal{H}_{\alpha}^{\mu}$ and $\omega \in \mathcal{L}^{1}(\mathbb{G})$,

$$
\begin{aligned}
(\omega \otimes \iota) \alpha(x) & =\Phi_{\alpha}^{\omega}(x)=\Phi_{\alpha}^{\omega} \Phi_{\alpha}^{\mu}(x)=\Phi_{\alpha}^{\omega \star \mu}(x) \\
& =\Phi_{\alpha}^{\mu \star \omega}(x)=\Phi_{\alpha}^{\mu} \Phi_{\alpha}^{\omega}(x)=\Phi_{\alpha}^{\mu}((\omega \otimes \iota) \alpha(x)),
\end{aligned}
$$

which implies that $(\omega \otimes \iota) \alpha(x) \in \mathcal{H}_{\alpha}^{\mu}$, for all $\omega \in \mathcal{L}^{1}(\mathbb{G})$, hence $\mathcal{H}_{\alpha}^{\mu}=\mathbb{C} 1$, by Theorem 3.3. Also $\mathbb{G}$ is amenable by [15, Theorem 4.2].

The next corollary can be regarded as a generalization of [12, Theorem 4.3], [18, Theorem 1.10], and [15, Theorem 4.2].

Corollary 3.5. A locally compact quantum group $\mathbb{G}$ is amenable if and only if there exist a nondegenerate spread-out $\mu \in \mathcal{P}(\mathbb{G})$ and $\nu \in \mathcal{M}(\mathbb{G})$ such that the spaces of fixed points of the left convolution map by $\mu$ and the right convolution map by $\nu$ on $\mathcal{L}^{\infty}(\mathbb{G})$ coincide.

As another immediate corollary to Theorem 3.3 , we obtain one of the main results of [4, a dual Choquet-Deny theorem: if $\mathbb{G}$ is a cocommutative locally compact quantum group, then $\mathcal{H}_{\Gamma}^{\mu}=\mathbb{C} 1$ for every nondegenerate $\mu \in \mathcal{P}(\mathbb{G})$.

Next we restrict ourselves to the actions of compact type. A quantum version of the Choquet-Deny theorem for compact groups is proved in [15, Theorem 5.3]. In the following, we generalize this result to the case of quantum ergodic actions.

Translating to our setting, the Choquet-Deny theorem for compact (quantum) groups states the triviality of harmonic functions associated to the action of a compact (quantum) group on itself, which is a compact (quantum) space. We show that weaker compactness type conditions are enough for the triviality of $\mathcal{H}_{\alpha}^{\mu}$. First note that by [15, Theorem 5.3] and Corollary 2.2. if $\mathbb{G}$ is compact and $\alpha: \mathbb{G} \curvearrowright N$ is ergodic, then $\mathcal{H}_{\alpha}^{\mu}=\mathbb{C} 1$ for all nondegenerate $\mu \in \mathcal{P}(\mathbb{G})$.

A state $\Omega$ on $N$ is said to be $\mathbb{G}$-invariant if $(\omega \otimes \Omega) \alpha=\langle\omega, 1\rangle \Omega$ for all $\omega \in \mathcal{L}^{1}(\mathbb{G})$. Note that for a normal state $\nu \in N_{*}$, the $\mathbb{G}$-invariance means $\omega \phi_{\nu}=\left(\Phi_{\alpha}^{\omega}\right)_{*}(\nu)=$ $\langle\omega, 1\rangle \nu$ for all $\omega \in \mathcal{L}^{1}(\mathbb{G})$.

Theorem 3.6. Let $\alpha$ be an ergodic action of a locally compact quantum group $\mathbb{G}$ on a $\sigma$-finite von Neumann algebra $N$. If there exists a normal $\mathbb{G}$-invariant state on $N$, then $\mathcal{H}_{\alpha}^{\mu}=\mathbb{C} 1$ for all nondegenerate spread-out $\mu \in \mathcal{P}(\mathbb{G})$.

Proof. Suppose that $\nu_{0} \in N_{*}$ is a normal $\mathbb{G}$-invariant state on $N$. Further suppose that $\omega$ is a faithful normal state on $\mathcal{L}^{\infty}(\mathbb{G})$. Then it follows from Theorem 2.3 that $\nu_{0}=\omega \phi_{\nu_{0}}$ is faithful on $N$. Now, if $\mu \in \mathcal{P}(\mathbb{G})$ is nondegenerate, it follows from (2.2) that $\nu_{0} E_{\alpha}^{\mu}=\nu_{0}$. Hence, the completely positive map $E_{\alpha}^{\mu}$ is normal, and therefore $\mathcal{H}_{\alpha}^{\mu}=\mathbb{C} 1$, by Corollary 2.6 .

As a consequence, Theorem 3.6 implies that harmonic functions are trivial in the case of probability measure preserving actions of locally compact groups.

We finish with another immediate corollary of Theorem [3.6] in the setting of group actions on finite factors. 
Corollary 3.7. Let $\alpha: G \curvearrowright N$ be an ergodic action of a locally compact group $G$ on a finite factor $N$, and let $\mu$ be a nondegenerate probability measure on $G$. If $x \in N$ is such that

$$
\int_{G} \alpha_{g}(x) d \mu(g)=x
$$

then $x \in \mathbb{C} 1$.

Proof. One only needs to observe that the unique finite faithful trace $\tau$ on $N$ is $G$-invariant.

\section{REFERENCES}

[1] William Arveson, Asymptotic stability. I. Completely positive maps, Internat. J. Math. 15 (2004), no. 3, 289-312, DOI 10.1142/S0129167X04002284. MR2060791 (2005g:46124)

[2] William Arveson, The asymptotic lift of a completely positive map, J. Funct. Anal. 248 (2007), no. 1, 202-224, DOI 10.1016/j.jfa.2006.11.014. MR2329688(2008k:46199)

[3] Man Duen Choi and Edward G. Effros, Injectivity and operator spaces, J. Functional Analysis 24 (1977), no. 2, 156-209. MR0430809 (55 \#3814)

[4] Cho-Ho Chu and Anthony To-Ming Lau, Harmonic functions on groups and Fourier algebras, Lecture Notes in Mathematics, vol. 1782, Springer-Verlag, Berlin, 2002. MR.1914221 (2003i:43001)

[5] Edward G. Effros and Zhong-Jin Ruan, Operator spaces, London Mathematical Society Monographs. New Series, vol. 23, The Clarendon Press, Oxford University Press, New York, 2000. MR1793753 (2002a:46082)

[6] Renato Feres and Emily Ronshausen, Harmonic functions over group actions, Geometry, rigidity, and group actions, Chicago Lectures in Math., Univ. Chicago Press, Chicago, IL, 2011, pp. 59-71. MR2807829 (2012e:60011)

[7] Y. Guivarc'h and C. R. E. Raja, Recurrence and ergodicity of random walks on linear groups and on homogeneous spaces, Ergodic Theory Dynam. Systems 32 (2012), no. 4, 1313-1349, DOI 10.1017/S0143385711000149. MR2955316

[8] Masaki Izumi, Non-commutative Poisson boundaries and compact quantum group actions, Adv. Math. 169 (2002), no. 1, 1-57, DOI 10.1006/aima.2001.2053. MR.1916370 (2003j:46105)

[9] Masaki Izumi, Non-commutative Poisson boundaries, Discrete geometric analysis, Contemp. Math., vol. 347, Amer. Math. Soc., Providence, RI, 2004, pp. 69-81, DOI 10.1090/conm/347/06267. MR2077031 (2005e:46126)

[10] Marius Junge, Matthias Neufang, and Zhong-Jin Ruan, A representation theorem for locally compact quantum groups, Internat. J. Math. 20 (2009), no. 3, 377-400, DOI 10.1142/S0129167X09005285. MR2500076 (2010c:46128)

[11] Vadim A. Kaimanovich, Measure-theoretic boundaries of Markov chains, 0-2 laws and entropy, Harmonic analysis and discrete potential theory (Frascati, 1991), Plenum, New York, 1992, pp. 145-180. MR.1222456 (94h:60099)

[12] V. A. Kaŭmanovich and A. M. Vershik, Random walks on discrete groups: boundary and entropy, Ann. Probab. 11 (1983), no. 3, 457-490. MR704539 (85d:60024)

[13] Vadim A. Kaimanovich and Wolfgang Woess, Boundary and entropy of space homogeneous Markov chains, Ann. Probab. 30 (2002), no. 1, 323-363, DOI 10.1214/aop/1020107770. MR.1894110 (2003d:60152)

[14] Mehrdad Kalantar, Representation of left centralizers for actions of locally compact quantum groups, Internat. J. Math. 24 (2013), no. 4, 1350025, 10, DOI 10.1142/S0129167X13500250. MR3062965

[15] Mehrdad Kalantar, Matthias Neufang, and Zhong-Jin Ruan, Poisson boundaries over locally compact quantum groups, Internat. J. Math. 24 (2013), no. 3, 1350023, 21, DOI 10.1142/S0129167X13500237. MR3048010

[16] M. Kalantar and M. S. M. Maokhar, Harmonic functions of measurable transformation groups, preprint

[17] Johan Kustermans and Stefaan Vaes, Locally compact quantum groups (English, with English and French summaries), Ann. Sci. École Norm. Sup. (4) 33 (2000), no. 6, 837-934, DOI 10.1016/S0012-9593(00)01055-7. MR 1832993(2002f:46108) 
[18] Joseph Rosenblatt, Ergodic and mixing random walks on locally compact groups, Math. Ann. 257 (1981), no. 1, 31-42, DOI 10.1007/BF01450653. MR630645 (83f:43002)

[19] Zhong-Jin Ruan, On the predual of dual algebras, J. Operator Theory 27 (1992), no. 1, 179-192. MR 1241122 (94k:47070)

[20] Stefaan Vaes, The unitary implementation of a locally compact quantum group action, J. Funct. Anal. 180 (2001), no. 2, 426-480, DOI 10.1006/jfan.2000.3704. MR.1814995 (2002a:46100)

Department of Mathematics, Tarbiat Modares University, Tehran 14115-134, Iran

E-mail address: mamini@modares.ac.ir

School of Mathematics and Statistics, Carleton University, Ottawa, Ontario, CANADA K1S 5B6

E-mail address: mkalanta@math.carleton.ca

Current address: Institute of Mathematics of the Polish Academy of Sciences, ul. S̀niadeckich 8, 00-956 Warszawa, Poland

E-mail address: mkalantar@impan.pl

Department of Mathematics, Tarbiat Modares University, Tehran 14115-134, Iran

E-mail address: m.mojahedi@modares.ac.ir 